\title{
Guest Editorial \\ Introduction to the Focused Section on Electromagnetic Devices for Precision Engineering
}

\section{INTRODUCTION}

$\mathbf{E}$ LECTROMAGNETIC devices have become more and more important in ultrahigh precision manufacturing, manipulation, and sensing due to their capability to provide large displacement with high positioning resolutions, apply multiaxis forces and torques, possess high bandwidth, and achieve reliability and versatility in applications. With wide availability of permanent magnetic materials and manufacturing technology, many new electromagnetic actuators and sensing elements have been explored in recent years. Applications of these electromagnetic devices have been seen often in micro- and nanomanufacturing, and ultrasensitive biomedical imagining systems. Additionally, these electromagnetic devices play crucial roles as the key components in renewable energy power generators as well as the driving elements of environmentally friendly electrical vehicles. In the realization of these novel electromagnetic devices, it is critical to have new groundbreaking modeling of electromagnetic components, integrated design of electromagnetic components with ultrahigh precision mechanisms, and precision control of the electromagnetic devices.

This "Focused Section on Electromagnetic Devices for Precision Engineering" of the IEEE/ASME TRANSACTIONS ON MECHATRONICS (TMECH) is dedicated to the new advances in modeling, design, analysis, control, and validation of electromagnetic devices for precision engineering. At the initial submission stage, a total number of 46 papers were received for the Focused Section. After going through a rigorous peer-review process with the help from the guest editors and numerous reviewers, 13 papers were invited for resubmission. These resubmitted papers went through a second round of peer view and finally nine papers were accepted and published in this Focused Section. The final papers were selected based on their merits, ingenuity, and relevance to this topic. It is our hope that the articles in this Focused Section can stimulate further research and technology breakthrough in this area as well as open up niche applications.

\section{Highlights OF THE FocUSEd Section AND OtHeR ELECTROMAGNETIC DEVICES PRESENTED IN THE TRANSACTIONS}

Electromagnetic devices, such as electromagnetic actuators and sensors, have a long history of development, and are still the main interests of researchers and engineers. The TRANSACTIONS archived numerous efforts on developing, validating, and

Digital Object Identifier 10.1109/TMECH.2011.2131147 deploying novel electromagnetic actuators, sensors, and systems for a very wide spectrum of applications. In this Editorial, we will highlight important papers related to this topic previously published in the TMECH and also in other journals. At the same time, we give an overview to the nine papers published in this Focused Section. It is interesting to note that in all the papers related to electromagnetic devices in TMECH, of which over $90 \%$ are directly related to the actuator design or actuation system design. As actuators are active elements that can produce work for different applications, they are among the main focuses in the engineering systems. In addition to actuators, sensors are also very critical components for feedback control and system integration. Magnetic levitation (maglev) systems, as a very important subtopic of electromagnetic devices, play a crucial role in precision engineering. Hence, the topic order of presentation in this Focused Section is precision actuators, precision sensors, novel maglev systems, and precision control of electromagnetic systems.

\section{A. Precision Electromagnetic Actuators}

High-precision motion control at nanometer scale is highly desirable in various industries and scientific applications. Awabdy et al. [1] realized a positioning resolution of $\pm 1 \mathrm{~nm}$ along 1-D by incorporating a secondary actuator on a standard linear motion table. This auxiliary device is a voice-coil actuator (VCA) capable of achieving 1-nm resolution at a maximum range of $2 \mu \mathrm{m}$. Dario et al. [2] addressed the need by proposing a new electromagnetic wobble micromotor. The actuator has a volume of $1 \mathrm{~cm}^{3}$ comprising of two wobble micromotors. The micromotor generates a torque of $350 \mu \mathrm{N} \cdot \mathrm{m}$ at each step and a maximum speed of approximately $180 \mathrm{r} / \mathrm{min}$. The application of such microactuators will form the basic components of high-precision micromechatronics system.

Petit et al. [3] proposed an electromagnetic actuator based on the digital principle in order to overcome the power and control challenges faced in the analogical actuators. The prototype has four discrete positions with an overall dimension of $25 \mathrm{~cm}^{2}$. It is able to produce $1 \mathrm{~mm} \times 1 \mathrm{~mm}$ stroke length at driving current from 3 to $7 \mathrm{~A}$. The advantage of such a digital actuator promises highly repeatable and accurate positions at the ends of each stroke. The byproduct is the sensorless design that simplifies electrical connections and the integration of the actuators in meso- or micromechanical systems for high-precision applications.

In the design of electromagnetic actuators, the description of magnetic field distribution is of paramount importance. $\mathrm{Hu}$ 
et al. [4] addressed this critical design aspect of electromagnetic mechatronic system by offering a cost-effective method in the computation of magnetic field distribution. The approach relaxes the assumptions made from known magnetic structures and calculates the field distribution based on the normal component of the magnetic flux density. Experimental validation was conducted on spherical and circular structures showing good agreement. The ability to reconstruct accurate magnetic field distribution will improve the performance of electromagnetic devices for subsequent implementation.

$\mathrm{Hu}$ and Kim [5] realized a high-precision positioner using a novel superimposed concentrated permanent magnet matrix. The device is capable of generating 6-DOF motions with only a single moving part. It comprises of three three-phase linearlevitation motors. Experimental data showed a position resolution of $20 \mathrm{~nm}$ with a position noise of $10-\mathrm{nm}$ rms in both $x$ - and $y$-directions. The positioning error in tracking a $1-\mu \mathrm{m}$ sinusoidal wave and a $5-\mu \mathrm{m}$ radius circle was less than $30-\mathrm{nm} \mathrm{rms}$, which demonstrated the positioner's 1-D and 2-D nanoscale positioning capabilities. Another 6-DOF precision magnetic actuator was evolved from Ren et al. [6] in which two self-bearing motors were employed to produce both radial bearing forces and motor torque. An active magnetic bearing was utilized to provide axial support and thus achieves a complete electromagnetic suspension and precision noncontact pointing. Experimental data show that the actuator is able to achieve a high angular resolution of 754 nrad over a large azimuth range of $\pm 45^{\circ}$.

Moving into the class of microelectromagnetic actuator, Komori and Hirakawa [7] developed a linear microactuator based on a new driving method. It composes of a mobile micro platform weighing $0.15 \mathrm{~g}$ and permanent magnets at a dimension of $8.5 \mathrm{~mm} \times 8.5 \mathrm{~mm} \times 0.1 \mathrm{~mm}$. The reported maximum displacement was $1.81 \mathrm{~mm}$. An immediate application for this microactuator is the microconveyer system.

\footnotetext{
"Radially biased axial magnetic bearings/motors for precision rotary-axial spindles," I. Usman, M. Paone, K. Smeds, and X. Lu from the University of British Columbia, Vancouver, BC, Canada.
}

Unlike traditional position holding thrust magnetic bearings, electromagnetic actuators can be advantageously used as both an axial bearing and an in-feed motor in precision machine tools that require only millimeter axial stroke [6]. In particular, active magnetic bearings, which are characterized by contactless support, are very successful in two application classes that are high-speed rotary machines and nanoscanning stages [8], [9].

For precision machine tools, a rotary spindle stage for cutting is typically installed on a linear $z$-stage for axial feeding. This serially stacked machine architecture suffers from stiffness reduction and precision degradation. When the in-feed stroke is at the millimeter level, magnetic bearings can be advantageously used as both a thrust bearing for the rotary spindle and a linear actuator for axial feeding. Indeed, radially biased axial bearing/motors have numerous advantages over other types of electromagnetic actuators, as higher load capacity, lower power dissipation, and smaller required armature. In addition, the coil windings can be moved away from armature pole surfaces to easily accommodate large coils windings for high-force generation.
Radially biased actuators have a fully linear force characteristic over the entire axial stroke. These performances characteristics make them excellent candidates in applications requiring both high-precision and high-load capacity [9].

This research group then deals with the design and the analysis of radially biased electromagnetic magnetic actuators for precision rotary-axial spindle applications. The overall rotaryaxial spindle design consists of journal bearings for radial support, a brushless motor for rotary driving, and an axial magnetic bearing/actuator. The experiments on a full-sized rotary-axial spindle showed that the radially biased actuator design achieves eight times higher load capacity and 16 times higher force-topower ratio than conventional horseshoe-type thrust bearings.

\footnotetext{
"Design and modeling of a 3-D magnetic actuator for magnetic microbead manipulation," Z. Zhang and C.-H. Menq from The Ohio State University, Columbus.
}

This research group presents a very interesting and novel hexapole 3-D magnetic actuator, wherein six electromagnets and six tip-shaped poles are used to generate magnetic field gradients and to exert noncontact magnetic forces on microscopic magnetic particles to allow back-and-forth actuation in three orthogonal directions. Since the motion of a magnetic particle placed in a magnetic field is inherently unstable without feedback control, additional means that achieve stabilization need to be employed to enable the magnetically propelled particle to act as a force-sensing probe. Two possible approaches have been proposed earlier. First, the magnetic particle is functionalized and anchored to target a biological sample to achieve stabilization [10], [11]. The actuator together with the functionalized magnetic particle then forms a magnetic tweezers [12]-[14], which is a simple force applier with an open-loop control, i.e., magnetic forces of desired magnitudes and directions are applied, and the induced motions of the magnetic particles are recorded by suitable measurement methods [15]. Second, an active feedback control is implemented to stabilize the magnetic particle by effectively changing the field potential according to the particle's motion measured in real time using a 3-D particle tracking technique. In this approach, the active feedback control achieves two objectives: 1) to stabilize the magnetically propelled particle; and 2) to rapidly propel the particle within the 3-D workspace to reach target locations and secure the wanted probe-sample engagement.

A multipole magnetic actuator is an over-actuated nonlinear system, wherein the number of input currents is more than that of output force components, and the resulting magnetic force is nonlinearly related to the input currents and has significant spatial heterogeneity. A quadrupole lumped-parameter force model was previously derived for a quadrupole magnetic actuator [16], and a model-based feedback control law was successfully established to realize stabilization and manipulation of the magnetic particle in a 2-D plane [17]. In this paper, a hexapole analytical model was derived for the 3-D magnetic actuator to accurately characterize the nonlinearity of the magnetic force exerting on the magnetic particle with respect to the applied currents and the position dependency of the magnetic force in the 3-D workspace. The authors then employed the 
analytical model to explore the force generation capability of the 3-D magnetic actuator. Moreover, a simplified inverse model associated with the lumped-parameter analytical force model was derived to determine the needed input currents that result in the desired magnetic force. Since the number of input currents is six and that of the output force components three, linear constraints among input currents and first-order approximation in spatial distribution were introduced to yield an analytical inverse model. This inverse model lays the foundation for current allocation and facilitates the implementation of feedback linearization. Experimental results in terms of magnetic force, in relation to the stable motion control of a magnetic particle were employed to validate the force model.

As a force applier, the 3-D magnetic actuator can investigate a wide range of biophysical properties, ranging from manipulating biological macromolecules [10], [11], [14], probing cell membranes [12], [18], to characterizing intracellular properties [19]-[21]. As a manipulator, it can steer a magnetic particle, serving as a measurement probe, to enable 3-D scanning probe microscopy.

\footnotetext{
"Bearingless permanent-magnet motor with 4/12 slot-pole ratio for bioreactor stirring applications," T. Reichert, T. Nussbaumer, W. Gruber, and J. W. Kolar from ETH Zurich and Levitronix GmbH, Zurich, Switzerland, and ACCM GmbH, Linz, Austria.
}

This research group presents the design and prototyping of a bearingless permanent magnet motor with very promising applications in biomedical engineering. The novel topology of the motor consists of an exterior disk-shaped rotor with six pole pairs and a stator with four stator teeth. The six pole pairs consist of twelve permanent magnets that are affixed to the back iron ring of the rotor and magnetized in alternating order. The input currents of the four concentrated stator coils simultaneously produce motor torque to control the speed of the rotor as well as bearing forces to stabilize the 5 remaining DOF. This motor is to be employed in delicate bioreactor processes. As the rotor is magnetically levitated, the motor is free of wear and thus, makes its lifespan long and the maintenance costs low [8].

Since the rotor has a disk shape, both axial and tilting displacements are stabilized passively by means of attracting reluctance forces [22], whereas stabilization of the radial displacements in $x$ - and $y$-directions requires an active feedback control. In this work, the relationship between the motor torque and the drive currents and that between the bearing forces and the input bearing currents are established. It is shown that the resulting torque/force is linearly related to the associated input currents, but its angular position dependent. They are then used to validate the topology of the motor, wherein the required motor torque and bearing forces can be independently realized by adjusting the four input currents.

For the purpose of feedback control, angular and displacement sensors are employed to measure the angular position and radial position of the rotor in real time. The control algorithm uses the radial position of the rotor to determine the required bearing force, and then allocates the four bearing currents according to the angular position of the rotor. It is also shown that the bearing currents do not influence the motor torque. In other words, the required input currents can be examined separately for motor torque and bearing forces, whereas they can be superimposed in the control commands to drive the same set of four coils. This concept of combined coils, i.e., no separate drive and bearing coils, is advantageous in terms of reducing the total required current, and hence, reducing copper losses [23].

With the topology of the motor, the authors used 3-D finite element method (FEM) analysis to determine the optimal sizing parameters of the motor to achieve two goals: 1) to realize maximal motor torque for performance and 2) to provide adequate bearing forces for stabilization. A prototype was built to validate the design. It uses concentrated, combined windings along with precise current allocation in a feedback control scheme to realize a high-performance bearingless motor in a very compact setup.

\section{B. Precision Electromagnetic Sensors}

Electromagnetic sensors are normally used in scenarios requiring the detection of magnetic fields. Sensors based on giant magnetoresistance [24] as well as anisotropic magnetorestrictive effects, and Hall and 3-D Hall sensors [25] have been served for this purpose in many industrial and precision devices applications. In order to precisely measure position and orientations using magnetic sensors, previous efforts require a direct mechanical linkage between the magnetic sensor and the device, such as an encoder enclosure. Due to the development of fast computation and embedded signal processing techniques, it is now possible to use magnetic sensors to measure and analyze the intricate magnetic fields so as to inversely derive the position and orientation in real time.

\footnotetext{
"Optimization of measuring magnetic fields for position and orientation tracking," W. Fang and H. Son from Nanyang Technological University, Singapore.
}

In recent years, actuator design is moving into putting multiple-DOF actuation into a single compact device. The development of electromagnetic 3-DOF spherical motors is a good example [56]. Such an actuator requires the design and packaging of multiple permanent magnetic poles and coils along a spherical geometry surface. The most challenging task now is the design of compact sensors capable of detecting multi-DOF motion for feedback control of the actuator. Existing precision position or orientation sensors can detect one-DOF motion only. To use them in multi-DOF systems, it requires additional mechanical structure support to achieve multi-DOF sensing. A laser displacement sensor-based technique to detect 2-DOF-orientation change has developed for such multi-DOF actuator [26]. However, the cost of the laser displacement sensor is too high to realize the wide application of the new actuator system. Hence, there is an urgent requirement for new multi-DOF motion sensor designs.

This paper introduced an interesting method to estimate the relative position and orientation of a magnetic sensor in 3-D space with respect to a cylindrical-shaped permanent magnet in motion. It is known that the 3-D magnetic field of a cylindrical permanent magnet, when rotating about a point in space, measured by a three-axis magnetic sensor at a fixed 
location forms an ellipsoid. The geometry of the ellipsoid is not only determined by the parameters of the magnet but also by the relative position and orientation between the sensor and the magnet. Thus, with known parameters of the magnet, its orientation and the position can be inversely computed using the magnetic field strength detected by the magnetic sensors. The authors utilize distributed multipole model to accurately characterize magnetic field of a cylindrical-shaped magnet as it is commonly seen in the electromagnetic actuator design. This method provides not only a closed form solution, but also has a very good performance accounting for the shape and size of the physical magnet. A genetic algorithm-based optimization has been used to determine the location and orientation of the sensor to maximize the measurement performance. The simulation and experimental results show the effectiveness of the method along with its ability to characterize the magnetic fields and compute the position/orientation, which offers a number of advantages in real-time measurement and control applications for multi-DOF actuators with multiple moving magnetic poles.

\section{Novel Maglev Systems}

Maglev is a promising technology in precision-positioning and transportation application. The maglev technology has been demonstrated successfully for nanopositioning applications. Several research groups developed precision positioning devices using this technology. Shan et al. [27], Holmes et al. [28], Hajjaji and Ouladsine [29], and Verma et al. [30] have done pioneering work in high-precision maglev. In the transportation applications, several maglev trains based on superconducting and traditional actuation mechanisms have been designed and tested [31]-[33]. The electromagnetic guiding system found in a maglev train can also be used in magnetic bearings and wind tunnels.

Karutz et al. [37] proposed a concept coined as magnetically levitated two-level motor (ML2M). Essentially, a homopolar bearing unit was separated axially from a multipolar drive unit at two different height levels. With this configuration, the motor torque was increased with high acceleration while still maintaining a compact structure.

The development of precision nanosystems demands for precise manufacturing of nanoparts, and maglev technology provides an avenue for this manipulation at the nanoscale level. Addressing this requirement, Verma et al. [30] proposed a 6-DOF magnetic levitated system that can achieve better than 5-nm resolution with $2-\mathrm{nm}$ rms position noise. It can reach a velocity of $0.5 \mathrm{~m} / \mathrm{s}$ and an acceleration of $30 \mathrm{~m} / \mathrm{s}^{2}$. The maximum payload was reported to be $1 \mathrm{~kg}$. Extending the range of the magneticlevitated stage, Kuo et al. [34] discussed on the use of an indirect adaptive-control approach to realize large travel ultraprecision $x-y-\theta$ motion. The gathered results demonstrated the superiority in tracking of the indirect adaptive controllers with an error of $\pm 10 \mathrm{~nm}$, over the constant gain robust linear $H^{\infty}$-controllers. Chen et al. [35] presented a novel dual-axis repulsive maglev guiding system that is also able to accomplish a larger motion range. The general framework for ultraprecision and large range motion control of magnetic levitated actuation system was sum- marized by Shan et al. [27], [36]. Most recently, Choi et al. [70] developed a high-precision dual-servo stage with a magnetically levitated fine stage. A Halbach linear active magnetic bearing (HLAMB) is used for maglev. The HLAMB utilizes permanent magnet arrays and Lorentz coils. The coarse stage has an $H$-type structure that can carry the fine stage $300 \mathrm{~mm} \times 300 \mathrm{~mm}$ along the $x$ - and $y$-axes. The experiment showed that it has 10 - and 15 -nm in-position stability in the $x$ - and $y$-axes, respectively.

\begin{abstract}
"Design and implementation of a new six-DOF maglev positioner with a fluid bearing," M.-Y. Chen, S.-C. Huang, S.-K. Hung, and L.-

C. Fu from National Taiwan Normal University and National Taiwan University, Taipei, Taiwan.
\end{abstract}

High-precision positioning systems are vital to a variety of relevant high-tech applications such as: surface IC- photolithography; multimedia home appliances (VCD/DVD players); electronic storage devices [hard disk drives (HDDs)]; and a multitude of scientific equipment (i.e., scanning probe microscopy). In this context, conventional positioning systems are facing severe challenges to achieve both high-resolution and large traveling range, along with additional requirements such as compact design and low-power consumption [38], [39]. In order to meet these challenges, more advanced positioning systems need to be developed.

In this context, the platform design is generally a tradeoff between resolution and travel range. Therefore, using noncontact forces generated between the carrier and the stationary platform seems to be a better solution in the aforementioned circumstances. Indeed, due to the noncontact property of maglev technology, the resulting system will exhibit some key features such as no friction, no contamination, long life, high speed, low noise, etc. [37].

This research group proposes an innovative 6-DOF maglevprecision positioner with fluid bearing capable of large motion range. Low-power consumption is achieved by using fluid buoyancy. Due the multiinput and multioutput nature of the developed system with nonnegligible modeling uncertainties, a robust adaptive sliding-mode controller has been developed [40]. The experimental results show that the proposed positioner is effective. Indeed, the positioning performances have reached the sensor resolution limit.

"Bilateral macro-micro teleoperation using magnetic levitation," M. Mehrtash, N. Tsuda, and M. B. Khamesee from the University of Waterloo, Waterloo, ON, Canada and Wakayama National College of Technology, Gobo, Japan.

This research group has bridged the manipulator and manipulated objects by developing a novel haptic magnetic levitation device (HMLD), consisting of a microslave robot, a macromaster robot, and a teleoperation system. HMLD allows a human operator to control the macroslave robot through manipulating the macromaster haptic robot. Moreover, the perception capability of the environment has been preserved in HMLD as well during the microdomain task operation. In order to improve the perception capability, the authors have used the position-error-based bilateral teleoperation. Hence, the position can be measured using external laser micrometer beams. The authors have analyzed the environment perception capability and the teleoperation, and 
afterward designed a gain-switching controller for system control. The experiment shows that the microrobot can follow the scaled hand motion with a high precision.

\section{Precision Control of Electromagnetic Systems}

To realize accurate positioning responses in any motion system, the control algorithm is an important factor on the achievable precision. Mittal and Menq [41] presented a robust nonlinear compensation approach to obtain large travel in magnetic suspension systems. The algorithm for compensation of unmatched uncertainties in feedback-linearized systems based on discrete-time delay control was investigated. Test results show that the controller is able to stabilize the system with an accuracy of $\pm 0.0127 \mathrm{~mm}$ and a tracking accuracy of $\pm 0.0381 \mathrm{~mm}$. It was concluded that the algorithm was able to accomplish a large travel range in spite of parameter variation and external disturbances. Jeong and Lee [42] on the other hand explored a time delay controller with state feedback for azimuth motion control of a magnetic suspension system. It was highlighted that the use of the low-pass filter increases the gain and phase margins and decreases the destabilization effect. Case studies supported the claim.

Aghili et al. [43] presented an integrated direct-drive system for high-precision motion control. The torque, motion and feedback aspects were addressed in the work. Empirical data show excellent performance in terms of thermal response, torque ripple, trajectory tracking, torque rejection, and joint stiffness. Chen et al. [44] looked into a learning scheme for low-speed precision tracking control of hybrid electromagnetic motors. The principles of learning control were employed to minimize the torque ripple on low-speed situations. It was observed that the proposed learning control scheme was able to drive tracking error to zero in the presence of unknown ripple dynamics.

Kuo and Menq [45] presented a six-axis magnetic suspension stage for precision motion control. Motion control was achieved via a parameter variation model in conjunction with a reduced order observer to compensate the combined effect of disturbances, modeling errors, and cross coupling. Tests validated the positioning stability and invariant dynamic response within the designated travel volume.

Gan and Cheung [46] developed a precision manufacturing system based on variable reluctance actuation principle. The highlight of the work is that the construction of the directdrive system requires no permanent magnets. Empirical data reveal that the tracking dynamic error is approximately $180 \mu \mathrm{m}$ with steady error below $20 \mu \mathrm{m}$. The work encompasses an effective nonlinear control method based on cascade structure and a nonlinear lookup table, avoiding complex mathematical functions. In the option of using conventional linear motors for high-precision applications, significant efforts have been devoted to solving the difficulties in controlling linear motors. $\mathrm{Xu}$ and Yao [47] studied and developed an adaptive robust controller that takes into account the effect of model uncertainties coming from inertia load, friction, force ripples, and external disturbances.
"Cascade modeling and intelligent control design for an electromagnetic guiding system," J.-D. Lee and R.-Y. Duan from National Formosa University, Yunlin, Taiwan, and Hung Kuang University, Tai Chung, Taiwan.

This research group proposes cascading modeling and intelligent control design for an electromagnetic guiding system that could be used in a maglev train. The guiding system consists of two EI-type electromagnets facing with each other across a linear guideway. With this guiding system, one lateral DOF of a maglev train can be controlled. The electromagnetic actuation forces were derived, and the electromagnets were modeled with a lumped-parameter electric circuit. The independent power module of the control system contains a pulsewidth modulation amplifier with the buck converter operated in the continuousconduction mode. The gaps between the linear guideway and the two electromagnets are controlled by a microcontroller with a bipolar switching device. The cascade sliding-mode control (SMC) system was derived using the Lyapunov method. This cascade SMC is embedded in a genetic algorithm, and a fuzzylogic controller was also developed based on the membership functions.

The cascade SMC and sliding-mode-based genetic algorithm control systems were first implemented in MATLAB with two simulation results. The authors also constructed a prototype maglev guideway test bed. In this test setup, two EI-type electromagnets are employed along with inductive gap sensors to measure the air gaps. A dual-loop control approach was adopted with the outer loop for the gap control and the inner loop for the current control. Experimental results exhibiting the guiding systems responses to a $2.5-\mathrm{mm}$ step signals were shown in the paper.

"Optimal reset control for a dual-stage actuator system in HDDs," H. Li, C. Du, and Y. Wang from Nanyang Technological University and the Data Storage Institute, Singapore.

HDDs are probably the most widely used commercial electromagnetic devices. Traditionally, a VCA with a single servo loop served as the key actuation system to seek and follow the tracks on the magnetic disk. One of the key technological limitations of VCAs is its low bandwidth, which prevents fast positioning of the read-write head thereby limiting its data-access speed. As the track density of an HDD increased dramatically to accommodate more data per unit area than earlier, dual-stage actuators (DSAs) have been introduced since the 1990s [38], [48]-[51]. In these DSAs, piezoelectric actuators or microelectromechanical systems-based actuators are employed to generate highprecision motions, and recently the piezoelectric actuator has become a workhorse in precision engineering with various applications [52]-[55].

This research group presents an optimal reset control (ORC) method that can be applied to a DSA in HDDs. The DSA specifically considered in this research consists of a VCA as the coarse-motion stage actuator and a push—pull PZT piezoelectric actuator as the fine- motion stage actuator. One of the technical challenges is that only the sum of the VCA and the PZT actuator outputs are available for the controller. Addressing this problem, the authors developed two ORCs for both the 
VCA and PZT stages. A performance index is utilized in the controller to convert this problem into a typical optimal control problem. The main contribution of this paper lies in the improvement of the control performance for a DSA in HDDs with fast track seeking. The controller follows a linear design with a fast rise time in the order of a faction of a microsecond, achieving a $40-\mathrm{kHz}$ sampling rate with a real-time digital-signal- processing system. The reset interval was chosen to be twice the sampling period, i.e., $50 \mu$ s. With this DSA system, a $2 \%$ overshoot was achieved.

\begin{abstract}
"Dynamics compensation and rapid resonance identification in ultrasonic-vibration-assisted microforming system using magnetostrictive actuator," Z. Wang, Q. Zou, L. Faidley, and G.-Y. Kim from Iowa State University, Ames, and Rutgers University, Piscataway, NJ.
\end{abstract}

By introducing ultrasonic vibration in the microforming process, energy can be effectively reduced and the tool life can be remarkably prolonged. However, the fundamental mechanism of the ultrasonic vibration on the dynamics of the microforming process is still unclear. Moreover, the actuator and controller design method are primitive and far from satisfactory. All these constitute the challenges of microforming systems embedded with ultrasonic vibrations. This research group has developed a mechatronic system to achieve rapid identification and tracking of the resonant frequency during the magnetostrictiveactuator-based microforming process. The experimental case study demonstrates that the ultrasonic vibration amplitudes were sharply decreased across a large frequency range and the computational complexity of online identification is also remarkably reduced as well. In summary, this work clearly demonstrates a very good precision manufacturing applications using electromagnetic devices.

\section{FUTURE TRENDS}

As prime movers, electromagnetic actuators are going into compact multiple DOF design. The development of electromagnetic 3-DOF spherical actuators is a major milestone for new actuator design using electromagnetism [56]-[58]. Such an actuator can produce 3 -DOF rotational motions in a compact ball-joint like structure. The application of such multi-DOF actuators is meant to replace complex actuation systems using serially connected single-DOF actuators and the complex motion and power transmission mechanisms. Other existing and new multi-DOF actuator configurations are in the form of 2-DOF planar actuators [59] and 2-DOF rotary/linear cylindrical actuators.

In addition to position and orientation sensing, highly sensitive sensors capable of detecting very weak magnetic fields have a spectrum of applications ranging from small memory devices to biomedical applications. Among these are sensors based on the magnetoimpedance (MI) effect that is a sensitive realignment of a periodic magnetization in response to an external magnetic field. Devices like MI-effect sensors that have experimentally demonstrated an absolute resolution on the order of $10^{-10} \mathrm{~T}$ or better [60] offer the potential of high sensitivity at low cost. As MI effects have complex behavior, the design of high-sensitivity MI sensors requires a good understanding of both electrodynamics and micromagnetic effects on the MI. Interest in MI has generated a flurry of research, since the discovery of Giant MI (GMI) effects in amorphous ferromagnetic wires [61]-[63] and thin films [64] with small magnetic fields at relatively low frequencies in the early 1990s. More than 100 promising applications of GMI have been suggested in [65]. A recent study [66] has provided a modeling method of the magnetic field in an MI sensor element and an investigation of the effects of micromagnetic coupling on the MI effect for biomedical applications.

For precision engineering applications, most of the time the actuators are used along with additional structural elements to provide a very high positioning capability, a high force/torque output, or a combination of both for micro- or nanomanufacturing needs. There is a tendency to simplify the structural element design and to pursue a new structural model technique for ease of control. For example, a new dual magnet actuator system, flexure-based electromagnetic linear actuator, employed a new semianalytical technique to model the flexures based on both theoretical and experimental study in a compact form so as to provide a simple linear control model for the actuator system to achieve the simultaneous position and force control for nanoresolution motion. Such an actuation system has been applied to microbiomedical device manufacturing [67]-[69].

Due to the wide availability of magnetic materials and wellestablished studies in magnetism, research and development efforts in electromagnetic devices, such as actuators, sensors and actuation systems will continue to strive and flourish. Significantly, these devices have strong industrial relevance with existing and new applications demanding for high-precision performance. Nevertheless, they will also find their new roles in the niche industry sectors such as new energy and electrical vehicles.

\section{ACKNOWLEDGMENT}

The Lead Guest Editor would like to thank Dr. C. K. Lim for the assistance during the collection of previous papers and organization of the literature survey. The Guest Editors of this Focused Section would like to express their sincere appreciation to all the authors who submitted their manuscripts for publication in this Focused Section. Also, the Guest Editors want to acknowledge the efforts of the reviewers who provided their professional and constructive comments in a timely manner. Finally, the Guest Editors would like to thank the Editor-in-Chief, Prof. K.-M. Lee, and M. Raine for their support and guidance throughout the production of this Focused Section.

I-Ming CHEN, Lead Guest Editor

School of Mechanical and Aerospace Engineering

Nanyang Technological University

Singapore 639798

Mohamed El Hachemi BenbouzID, Guest Editor University of Brest

29238 Brest Cedex, France 


\author{
HAN DING, Guest Editor \\ National Laboratory of Digital Manufacturing \\ Equipment and Technology \\ Huazhong University of Science and Technology \\ Wuhan 430074, China
}

\section{WON-JONG KIM, Guest Editor}

Department of Mechanical Engineering

Texas A\&M University

College Station, TX 77843-3123 USA

\section{Chia-Hsiang MenQ, Guest Editor \\ Department of Mechanical Engineering \\ The Ohio State University \\ Columbus, OH 43210 USA}

\section{REFERENCES}

[1] B. A. Awaddy, W.-C. Shih, and D. M. Auslander, "Nanometer positioning of a linear motion stage under static loads," IEEE/ASME Trans. Mechatronics, vol. 3, no. 2, pp. 113-119, Jun. 1998.

[2] P. Dario, M. C. Carrozza, C. Stefanini, and S. D'Attanasio, "A mobile microrobot actuated by a new electromagnetic wobble micromotor," IEEE/ASME Trans. Mechatronics, vol. 3, no. 1, pp. 9-16, Mar. 1998.

[3] L. Petit, C. Prelle, E. Dore, F. Lamarque, and M. Bigerelle, "A fourdiscrete-position electromagnetic actuator: modeling and experimentation," IEEE/ASME Trans. Mechatronics, vol. 15, no. 1, pp. 88-96, Feb. 2010.

[4] L. Hu, K.-M. Lee, and X. Fu, "A method based on measured boundary conditions for reconstructing the magnetic field distribution of an electromagnetic mechatronic system," IEEE/ASME Trans. Mechatronics, vol. 15 , no. 4, pp. 595-602, Aug. 2010.

[5] T. J. Hu and W.-J. Kim, "Extended range six-DOF high-precision positioner for wafer processing," IEEE/ASME Trans. Mechatronics, vol. 11, no. 6, pp. 682-689, Dec. 2006.

[6] Z. Ren and L. S. Stephens, "Closed-loop performance of a six degree-offreedom precision magnetic actuator," IEEE/ASME Trans. Mechatronics, vol. 10, no. 6, pp. 666-674, Dec. 2005.

[7] M. Komori and T. Hirakawa, "A magnetically driven linear microactuator with new driving method," IEEE/ASME Trans. Mechatronics, vol. 10, no. 3, pp. 335-338, Jun. 2005.

[8] S. H. Park and C. W. Lee, "Decoupled control of a disk-type rotor equipped with a three-pole hybrid magnetic bearing," IEEE/ASME Trans. Mechatronics, vol. 15 , no. 5, pp. 793-804, Oct. 2010.

[9] C. Weissbacher, H. Stelzer, and K. Hameyer, "Application of a tubular linear actuator as an axial magnetic bearing," IEEE/ASME Trans. Mechatronics, vol. 15, no. 4, pp. 615-622, Aug. 2010.

[10] C. Haber and D. Wirtz, "Magnetic tweezers for DNA micromanipulation," Rev. Sci. Instrum., vol. 71, no. 12, pp. 4561-4570, 2000.

[11] J. Zlatanova and S. H. Leuba, "Magnetic tweezers: A sensitive tool to study DNA and chromatin at the single-molecule level," Biochem. Cell Biol., vol. 81, pp. 151-159, 2003.

[12] A. R. Bausch, W. Moller, and E. Sackmann, "Measurement of local viscoelasticity and forces in living cells by magnetic tweezers," J. Biophys., vol. 76, pp. 573-579, 1999.

[13] J. K. Fisher, J. Cribb, K. V. Desai, L. Vicci, B. Wilde, K. Keller, R. M. Taylor, II, J. Haase, K. Bloom, E. Timothy O'Brien, and R. Superfine, "Thin-foil magnetic force system for high-numerical-aperture microscopy," Rev. Sci. Instrum., vol. 77, 023702, 2006.

[14] C.-H. Chiou, Y.-Y. Huang, M.-H. Chiang, H.-H. Lee, and G.-B. Lee, "New magnetic tweezers for investigation the mechanical properties of single DNA molecules," Nanotechnol, vol. 17, pp. 1217-1224, 2006.

[15] Z. Zhang and C.-H. Menq, "Three-dimensional particle tracking with subnanometer resolution using off-focus images," Appl. Opt., vol. 47, no. 14 , pp. 2361-2370, 2008.

[16] Z. Zhang, K. Huang, and C.-H. Menq, "Design, implementation, and force modeling of quadruple magnetic tweezers," IEEE/ASME Trans. Mechatronics, vol. 15, no. 5, pp. 704-713, Oct. 2010.

[17] Z. Zhang, Y. Huang, and C.-H. Menq, "Actively controlled manipulation of a magnetic microbead using quadrupole magnetic tweezers," IEEE Trans. Robot., vol. 26, no. 3, pp. 531-541, Jun. 2010.
[18] Z. Zhang, Y. Shi, S. M. Jhiang, and C.-H. Menq, "Mechanical anisotropy and adaptation of metastatic cells probed by magnetic microbeads," presented at the Proc. SPIE- Int. Soc. Opt. Eng.; Conf. Imag., Manipulation, Anal. Biomolecules, Cells, Tissues VII, San Francisco, CA, Jan. 23-25, 2010.

[19] B. G. Hosu, K. Jakab, P. Banki, and F. I. Toth, "Magnetic tweezers for intracellular applications," Rev. Sci. Instrum., vol. 74, no. 9, pp. 4158 4163, 2003.

[20] A. H. B. de Vries, B. E. Krenn, R. van Driel, and J. S. Kanger, "Micro magnetic tweezers for nanomanipulation inside live cells," Biophys. J., vol. 88, no. 3, pp. 2137-2144, 2005.

[21] J. S. Kanger, V. Subramaniam, and R. van Driel, "Intracellular manipulation of chromatin using magnetic nanoparticles," Chromosome Res. vol. 16, pp. 511-522, 2008.

[22] Q. D. Nguyen and S. Ueno, "Modeling and control of salient-pole permanent magnet axial-gap self-bearing motor," IEEE/ASME Trans. Mechatronics, Apr. 2010. [Online]. Available: DOI 10.1109/TMECH. 2010.2045392

[23] K. Raggl, T. Nussbaumer, and J. W. Kolar, "Comparison of separated and combined winding concepts for bearingless centrifugal pumps," J. Power Electron., vol. 9, no. 2, pp. 243-258, 2009.

[24] M. M. Miller et al., "Development of a high-precision absolute linear displacement sensor utilizing GMR spin-valves," IEEE Trans. Magn., vol. 33, no. 5, pp. 3388-3390, Sep. 1997.

[25] R. S. Popovic, J. A. Flanagan, and P. A. Besse, "The future of magnetic sensors," Sens. Actuators A, vol. 56, pp. 39-55, 1996.

[26] L. Yan, I.-M. Chen, Z. W. Guo, Y. Lang, and Y. H. Li, "A three- degreeof-freedom non-contact orientation measurement methodology with laser detection," IEEE Trans. Autom. Sci. Eng., vol. 18, no. 2, pp. 319-326, 2011.

[27] X. Shan, S.-K. Kuo, J. Zhang, and C.-H. Menq, "Ultraprecision motion control of a multiple degrees of freedom magnetic suspension stage," IEEE/ASME Trans. Mechatronics, vol. 7, no. 1, pp. 67-78, Mar. 2002.

[28] M. Holmes, R. Hocken, and D. L. Trumper, "The long-range scanning stage: A novel platform for scanned-probe microscopy," Precision Eng., vol. 24, no. 3, pp. 191-209, Jul. 2000.

[29] E. Hajjaji and M. Ouladsine, "Modeling and nonlinear control of magnetic levitation systems," IEEE Trans. Ind. Electron., vol. 48, no. 4, pp. 831838, Aug. 2001.

[30] S. Verma, W.-J. Kim, and J. Gu, "Six-axis nanopositioning device with precision magnetic levitation technology," IEEE/ASME Trans. Mechatronics, vol. 9, no. 2, pp. 384-391, Jun. 2004.

[31] S. Sawada, "Outlook of the superconducting Maglev," Proc. IEEE, vol. 97, no. 11, pp. 1881-1885, Nov. 2009.

[32] L. Yan, "Progress of the maglev transportation in China," IEEE Trans. Appl. Supercond., vol. 16, no. 2, pp. 1138-1141, Jun. 2006.

[33] D. Y Park, B. C. Shin, and H. Han, "Korea's urban maglev program," Proc. IEEE, vol. 97, no. 11, pp. 1886-1891, Nov. 2009.

[34] S.-K. Kuo, X. Shan, and C.-H. Menq, "Large travel ultraprecision $\mathrm{x}-\mathrm{y}-$ $\theta$; motion control of a magnetic-suspension stage," IEEE/ASME Trans. Mechatronics, vol. 8, no. 3, pp. 334-341, 2003.

[35] M.-Y. Chen, M.-J. Wang, and L.-C. Fu, "A novel dual-axis repulsive Maglev guiding system with permanent magnet: Modeling and controller design," IEEE/ASME Trans. Mechatronics, vol. 8, no. 1, pp. 77-86, Mar. 2003.

[36] X. Shan and C.-H. Menq, "Robust disturbance rejection for improved dynamic stiffness of a magnetic suspension stage," IEEE/ASME Trans. Mechatronics, vol. 7, no. 3, pp. 289-295, Sep. 2002.

[37] P. Karutz, T. Nussbaumer, W. Gruber, and J. W. Kolar, "Novel magnetically levitated two-level motor," IEEE/ASME Trans. Mechatronics, vol. 13, no. 6, pp. 658-668, Dec. 2008.

[38] K. W. Chan, W. H. Liao, and I. Y. Shen, "Precision positioning of hard disk drives using piezoelectric actuators with passive damping," IEEE/ASME Trans. Mechatronics, vol. 13, no. 1, pp. 147-151, Feb. 2008.

[39] H. Yu and W.-J. Kim, "A compact Hall-effect-sensing 6-DOF precision positioner," IEEE/ASME Trans. Mechatronics, vol. 15, no. 6, pp. 982 985, Dec. 2010.

[40] J. H. She, X. Xin, and Y. Pan, "Equivalent-input-disturbance approach: Analysis and application to disturbance rejection in dual-stage feed drive control system," IEEE/ASME Trans. Mechatronics, vol. 16, no. 2, pp. 330340, Apr. 2011.

[41] S. Mittal and C.-H. Menq, "Precision motion control of a magnetic suspension actuator using a robust nonlinear compensation scheme," IEEE/ASME Trans. Mechatronics, vol. 2, no. 4, pp. 268-280, Dec. 1997. 
[42] H.-S. Jeong and C.-W. Lee, "Time delay control with state feedback for azimuth motion of the frictionless positioning device," IEEE/ASME Trans. Mechatronics, vol. 2, no. 3, pp. 161-168, Sep. 1997.

[43] F. Aghili, J. M. Hollerbach, and M. Buehler, "A modular and highprecision motion control system with an integrated motor," IEEE/ASME Trans. Mechatronics, vol. 12, no. 3, pp. 317-329, Jun. 2007.

[44] W. D. Chen, K. L. Yung, and K. W. Cheng, "A learning scheme for lowspeed precision tracking control of hybrid stepping motors," IEEE/ASME Trans. Mechatronics, vol. 11, no. 3, pp. 362-365, Jun. 2006.

[45] S.-K. Kuo and C.-H. Menq, "Modeling and control of a six-axis precision motion control stage," IEEE/ASME Trans. Mechatronics, vol. 10, no. 1, pp. 50-59, Feb. 2005

[46] W. C. Gan and N. C. Cheung, "Development and control of a low-cost linear variable-reluctance motor for precision manufacturing automation," IEEE/ASME Trans. Mechatronics, vol. 8, no. 3, pp. 326-333, Sep. 2003.

[47] L. Xu and B. Yao, "Adaptive robust-precision motion control of linear motors with negligible electrical dynamics: Theory and experiments," IEEE/ASME Trans. Mechatronics, vol. 6, no. 4, pp. 444-452, Dec. 2001.

[48] C. K. Pang, G. Guo, B. M. Chen, and T. H. Lee, "Self-sensing actuation for nanopositioning and active-mode damping in dual-stage HDDs," IEEE Trans. Mechatronics, vol. 11, no. 3, pp. 328-338, Jun. 2006.

[49] S. Koganezawa, Y. Uematsu, T. Yamada, H. Nakano, J. Inoue, and T. Suzuki, "Dual-stage actuator system for magnetic disk drives using a shear mode piezoelectric microactuator," IEEE Trans. Magn., vol. 35, no. 2, pp. 988-992, Mar. 1999.

[50] T. Semba, T. Hirano, J. Hong, and L. Fan, "Dual-stage servo controller for HDD using MEMS microactuator," IEEE Trans. Magn., vol. 35, no. 5, pp. 2271-2273, Sep. 1999.

[51] Y. Soeno, S. Ichikawa, T. Tsuna, Y. Sato, and I. Sato, "Piezoelectric piggyback microactuator for hard disk drive," IEEE Trans. Magn., vol. 35, no. 2, pp. 983-987, Mar. 1999.

[52] J. Ni and Z. Zhu, "Design of a linear piezomotor with ultrahigh stiffness and nanoprecision," IEEE/ASME Trans. Mechatronics, vol. 5, no. 4, pp. 441-443, Dec. 2000.

[53] P. E. Tenzer and R. B. Mrad, "A systematic procedure for the design of piezoelectric inchworm precision positioners," IEEE/ASME Trans. Mechatronics, vol. 9, no. 2, pp. 427-435, Jun. 2004.

[54] Y. Shen, E. Winder, N. Xi, C. A. Pomeroy, and U. C. Wejinya, "Closed-loop optimal control-enabled piezoelectric microforce sensors," IEEE/ASME Trans. Mechatronics, vol. 11, no. 4, pp. 420-427, Aug. 2006.

[55] S.-K. Hung, E.-T. Hwu, M.-Y. Chen, and L.-C. Fu, "Dual-stage piezoelectric nanopositioner utilizing a range-extended optical fiber fabry-perot interferometer," IEEE/ASME Trans. Mechatronics, vol. 12, no. 3, pp. 291298, Jun. 2007.

[56] L. Yan, I.-M. Chen, G. Yang, and K.-M. Lee, "Analytical and experimental investigation on the magnetic field and torque of a permanent magnet spherical actuator," IEEE Trans. Mechatronics, vol. 11, no. 4, pp. 409_ 419, Aug. 2006.

[57] L. Yan, I.-M. Chen, C. K. Lim, G. Yang, W. Lin, and K.-M. Lee, "Design and analysis of a permanent magnet spherical actuator," IEEE Trans. Mechatronics, vol. 13, no. 2, pp. 239-248, Apr. 2008.

[58] L. Yan, I.-M. Chen, C. K. Lim, G. Yang, and W. Lin, "Analysis of pole configurations of permanent magnet spherical actuators," IEEE/ASME Trans. Mechatronics, vol. 15, no. 6, pp. 985-989, Dec. 2010.

[59] W.-J. Kim, D. L. Trumper, and J. H. Lang, "Modeling and vector control of planar magnetic levitator," IEEE Trans. Ind. Appl., vol. 34, no. 6, pp. 1254-1262, Nov./Dec. 1998.

[60] K. Mohri, T. Uchiyama, and L. V. Panina, "Recent advances of micro magnetic sensors and sensing application," Sens. Actuators, vol. 59, pp. 1-8, 1997.

[61] K. Mohri, K. Kawashima, T. Kohzawa, and H. Yoshida, "Magnetoinductive element," IEEE Trans. Magn., vol. 29, no. 2, pp. 1245-1248, 1993.

[62] R. S. Beach and A. E. Berkowitz, "Giant-magnetic field dependent impedance of amorphous FeCoSiB wire," Appl. Phys. Lett., vol. 64, pp. 3652-3654, 1994.

[63] L. V. Panina and K. Mohri, "Magneto-impedance effect in amorphous wires," Appl. Phys. Lett., vol. 65, no. 9, pp. 1189-1191, Aug. 1994.

[64] F. L. A. Machado, B. L. da Silva, S. M. Rezende, and C. S. Martins, "Giant AC magneto-resistance in the soft ferromagnet $\mathrm{Co}_{75-x} \mathrm{Fe}_{x} \mathrm{Si}_{15} \mathrm{~B}_{10}$," Phys. Rev. B, vol. 51, pp. 3926-3929, 1995.

[65] K. Mohri, T. Uchiyama, L. P. Shen, C. M. Cai, and L. V. Panina, "Sensitive micro magnetic sensor family utilizing magneto-impedance (MI) and stress-impedance (SI) effects for intelligent measurements and controls," Sens. Actuators (Phys.), vol. A91, pp. 85-90, 2001.

[66] K. Eason and K.-M. Lee, "Effects of nonlinear micromagnetic coupling on a weak-field magnetoimpedance sensor," IEEE Trans. Magn., vol. 44, no. 8, pp. 2042-2048, Aug. 2008.

[67] T. J. Teo, I.-M. Chen, G. Yang, and W. Lin, "Magnetic field modeling of a dual-magnet configuration," J. Appl. Phys., vol. 102, 074924, 2007.

[68] T. J. Teo, I.-M. Chen, G. Yang, and W. Lin, "A flexure-based electromagnetic linear actuator," Nanotechnol., vol. 19, pp. 315501-315510, 2008.

[69] T. J. Teo, I.-M. Chen, G. Yang, and W. Lin, "Generic approximation solution for nonlinear deflection analyses of beam-based flexure joints," Precision Eng., vol. 34, no. 3, pp. 607-618, Jul. 2010.

[70] Y. M. Choi and D.-G. Gweon, "A high-precision dual-servo stage using halbach linear active magnetic bearings," IEEE/ASME Trans. Mechatronics, Aug. 2010. [Online]. Available: DOI: 10.1109/TMECH. 2010.2056694.

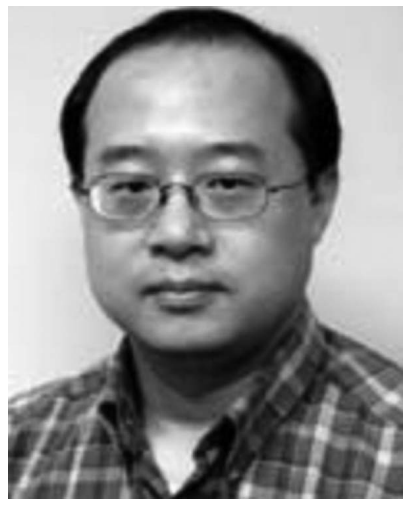

design.

Dr. Chen is a Fellow of the American Society of Mechanical Engineers (ASME) and a Senior Member of the IEEE Robotics and Automation Society. He was a Technical Editor of the IEEE/ASME TRANSACTIONS ON MECHATRONICS from 2003 to 2009. He is currently serving on the Editorial Boards of the IEEE TRANSACTIONS ON ROBOTICS, Mechanism and Machine Theory, and Robotica, and also the Associate Editor-in-Chief of Frontier of Mechanical Engineering (Berlin, Germany: Springer-Verlag). He was the General Chairman of the 2006 IEEE Conferences on Cybernetics, Intelligent Systems, and Robotics (CIS-RAM) in Thailand, and the 2009 IEEE/ASME International Conference on Advanced Intelligent Mechatronics (AIM 2009) in Singapore. He was the founding Chairman of the IEEE Robotics and Automation Singapore Chapter in 2001 and led the chapter to win the Best RAS Chapter Award in 2003. He is also the Chairman of the Technical Committee on Robotics and Mechatronics under the International Federation for the Promotion of Mechanism and Machine Science (IFToMM). 


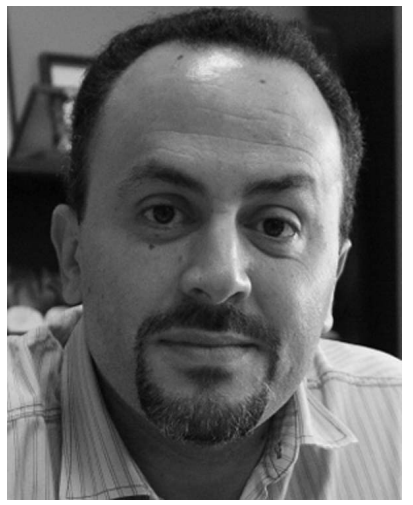

Mohamed El Hachemi Benbouzid (S'92-M'95-SM'98) was born in Batna, Algeria, in 1968. He received the B.Sc. degree in electrical engineering from the University of Batna, Batna, in 1990, the M.Sc. and Ph.D. degrees in electrical and computer engineering from the National Polytechnic Institute of Grenoble, Grenoble, France, in 1991 and 1994, respectively, and the Habilitation à Diriger des Recherches degree from the University of Picardie "Jules Verne," Amiens, France, in 2000.

After receiving the Ph.D. degree, he joined the Professional Institute of Amiens, University of Picardie "Jules Verne," where he was an Associate Professor of Electrical and Computer Engineering. In September 2004, he joined the University Institute of Technology (IUT) of Brest, University of Brest, Brest, France, as a Professor of Electrical Engineering. His current research interests and experience include analysis, design, and control of electric machines, variable-speed drives for traction, propulsion, and renewable energy applications, and fault diagnosis of electric machines.

Dr. Benbouzid is a Senior Member of the IEEE Power Engineering, IEEE Industrial Electronics, IEEE Industry Applications, IEEE Power Electronics, and IEEE Vehicular Technology Societies. He is an Associate Editor of the IEEE TRANSACTIONS ON ENERGY CONVERSION, IEEE TRANSACTIONS ON INDUSTRIAL ELECTRONICS, IEEE TRANSACTIONS ON VEHICULAR TECHNOLOGY, and IEEE/ASME TRANSACTIONS ON MECHATRONICS.

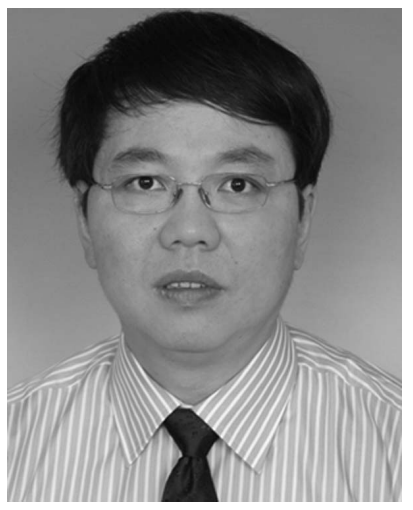

Han Ding (M'97-SM'00) received the Ph.D. degree from Huazhong University of Science and Technology (HUST), Wuhan, China, in 1989.

Supported by the Alexander von Humboldt Foundation, he was at the University of Stuttgart, Stuttgart, Germany, from 1993 to 1994. He was at the School of Electrical and Electronic Engineering, Nanyang Technological University, Singapore, from 1994 to 1996. He has been a Professor at HUST since 1997. He is the Director of the State Key Laboratory of Digital Manufacturing Equipment and Technology at HUST. He is also a "Cheung Kong" Chair Professor at Shanghai Jiao Tong University, Shanghai, China. His research interests include robotics , multiaxis machining, and equipment automation.

Dr. Ding is currently a Technical Editor of the IEEE/ASME TRANSACTIONS ON MECHATRONICS. He serves as a Guest Editor and a Technical Committee Member of Semiconductor Manufacturing Automation in the IEEE Robotics and Automation Society. From 2004 to 2007, he was an Associate Editor of the IEEE TRANSACTIONS ON AUTOMATION SCIENCE AND ENGINEERING. He was a CASE Local Chair in 2006. He is the General Co-Chair of the 2011 IEEE International Conference on Robotics and Automation in Shanghai. He has organized and chaired many technical sessions and workshops at various international conferences.

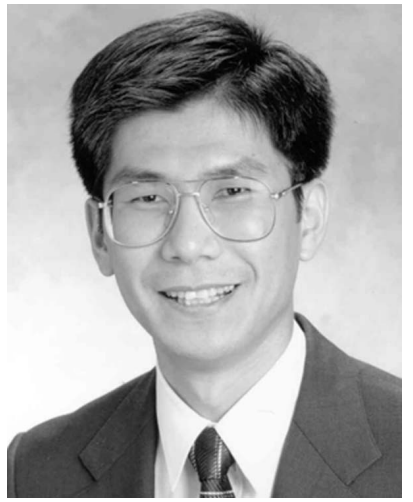

Won-jong Kim (S'89-M'97-SM'03) received the B.S. (summa cum laude) and M.S. degrees in control and instrumentation engineering from Seoul National University, Seoul, Korea, in 1989 and 1991, respectively, and the Ph.D. degree in electrical engineering and computer science from the Massachusetts Institute of Technology (MIT), Cambridge, in 1997.

Since 2000, he has been with the Department of Mechanical Engineering, Texas A\&M University, College Station, where he is currently an Associate Professor and was the inaugural holder of the Dietz Career Development Professorship II during 2007-2010. He is the holder of three U.S. patents on precision positioning systems. His current research interests include the analysis, design, and real-time control of mechatronic systems, networked control systems, and nanoscale engineering and technology.

Dr. Kim is a Fellow of the American Society of Mechanical Engineers (ASME) and a member of Pi Tau Sigma. He is a Technical Editor of the IEEE/ASME TRANSACTIONS ON MECHATRONICS, ASME Journal of Dynamic Systems, Measurement and Control, and International Journal of

Control, Automation, and Systems. 


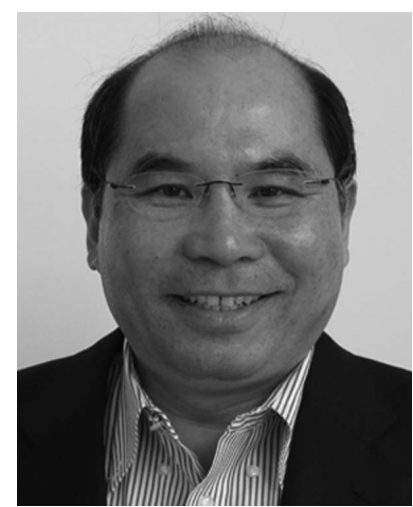

Chia-Hsiang Menq (M'03-SM'08) received the B.S. degree from National Tsing-Hua University, Taipei, Taiwan, in 1978, and the M.S. and Ph.D. degrees from Carnegie-Mellon University, Pittsburgh, PA, in 1982 and 1985, respectively, all in mechanical engineering.

Since 1985, he has been with The Ohio State University, Columbus, where he is currently a Professor and the Ralph W. Kurtz Chair in Mechanical Engineering. His research interests include: control, sensing, and instrumentation; metrology, precision engineering, and manufacturing; imaging and mechanical characterization of live cells; and visual sensing and visual servo control.

Dr. Menq is a Fellow of the American Society of Mechanical Engineers, the Association for the Advancement of Science, and the Society of Manufacturing Engineers. 